\title{
Vascular surgical and interventional services review: proposal to implement a regional vascular network and specialty vascular intervention centres Mark Tomlinson, FRCS
}

The North West is currently undergoing a regional review of vascular surgical and radiological services, with a plan to reduce the number of centres providing such inpatient services (whilst maintaining local outpatient and day-case intervention services), but increase or maintain patient access to modern vascular surgical services, improve available expertise, improve associated patient outcomes related to vascular interventions, and of course reduce costs.

This review has been split into three sub-regions: Cumbria and Lancashire; Greater Manchester; and Cheshire and Merseyside. The Cumbria and Lancashire review process is now at the stage where acute hospital trusts have been asked to submit expressions of interest to provide such services, ie to host one of the vascular intervention centres. This process is being mediated by the Strategic Health Authority and commissioning bodies; primary care trusts and now general practitioner (GP) cluster commissioners, with the assistance of the cardiac and stroke management team and the Cumbria and Lancashire Vascular Network Clinical Advisory Group.

The suggested clinical model is to centralise all inpatient arterial vascular interventions to the selected three acute trust centres, which would serve the entire population of Lancashire and Cumbria, to also include the populations of Wigan, Bolton and Leigh, and perhaps additionally Dumfries and Galloway in Scotland - thus giving a total population of 2.7 million. The vascular team at University Hospitals of Morecambe Bay (UHMB) have fully participated with impartial and positive contributions in the Vascular Network Clinical Advisory Group meetings held over the last 18 months, though these meetings specifically excluded discussion of any actual geographical sites or named institutions other than the issue of Galloway and Dumfries.

The review deadline for acute trusts within the region to express their interest is II November 20 I , after which the submissions will be assessed and the review decisions will, apparently, be announced by 5 January 2012, with a ten-day window for unsuccessful provider organisations to appeal.

As part of the review process local GPs throughout the region are being asked to complete an online survey regarding vascular services, hosted on a secure server and accessed at: www.feedbacksurveys.org.uk/vasculargp. This takes approximately five minutes to complete and is available until late November.

A public engagement process has also commenced with an online e-survey for any member of the public to participate, and for service users, prospective patients, carers, healthcare staff, and those with an interest in vascular services. This is available to anyone who is interested at: www.feedbacksurveys.org.uk/vascularservices . Paper copies of the questionnaire are available by telephoning 08456031068 again until late November. Please take the time to view and complete these surveys.

\section{BACKGROUND}

For some years, there has been increasing specialisation amongst surgeons and interventional radiologists performing invasive vascular procedures. This has been driven by a variety of factors: improved patient outcomes after vascular procedures with increased volume of procedures; better recognition of new or complex therapeutic options; adoption of less invasive procedures; increased use of radiological image guidance to perform procedures; perceived technical complexity of these new developments; need for adequate training in these aspects of interventional care; and withdrawal of non-vascular general surgeons and radiologists from providing emergency vascular interventions. The latter led to the formation of specific vascular emergency rotas provided by clinical networks formed by interested vascular surgeons (and radiologists) in adjacent hospitals. However, these have often been informal and ad hoc, of variable reliability, and thus geographical variations of service have existed with inequality of access and patient outcomes.

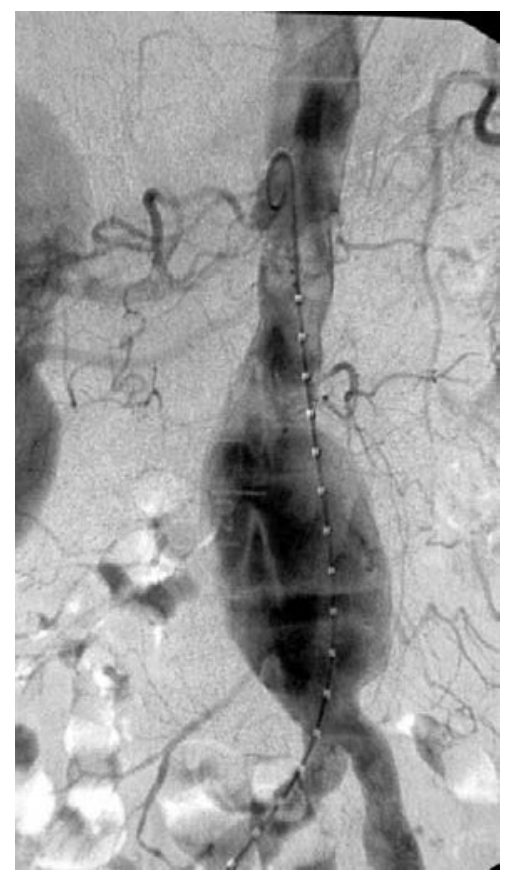

On-table angiogram showing an abdominal aortic aneurysm with a guide wire along which the endovascular prosthesis is inserted 


\section{NATIONALVASCULAR DEVELOPMENTS}

In America, and most of Europe, vascular surgery has been a separate surgical specialty for some years, (akin to cardiovascular surgery or neurosurgery, for example), whereas in the UK, it has remained a sub-specialty branch of general surgery, delivered in most acute hospitals. Over the last ten years, the Vascular Society of Great Britain and Ireland has lobbied the surgical Royal Colleges, General Medical Council and government to grant specialty status to vascular surgery, and this now looks likely to actually occur, though the timescale is still uncertain. Although provisionally agreed with the current government, the process still requires debate and support in the House of Lords.

Factors for centralisation of major arterial interventions are the provision of emergency vascular interventions, improving patient outcomes and recently, in particular, the implementation of a national abdominal aortic aneurysm screening programme (NAAASP) to identify abdominal aortic aneurysms (AAA) in males aged 65 years. The Vascular Society and NAASP have determined that all patients with screendetected aneurysms will undergo elective procedures in defined Quality Assured treatment centres, and these must achieve the National AAA Quality Improvement Programme outcome target of an overall maximum AAA treatment mortality rate of $3.5 \%$ (combined open and endovascular procedures) to ensure that the benefits of screening are achieved. Thus, over half of the AAA interventions will be endovascular aneurysm repair (EVAR) techniques to reduce the procedural 30-day mortality, and cost of AAA repairs by reduced inpatient length of stay. The number of AAA procedures performed per unit will be a minimum of 50 interventions per year to ensure sufficient unit expertise, and thus requires such units to serve a minimum population base of approximately 800,000 . In large urban conurbations, this is readily achievable with reasonable travel times, but will of course be more challenging in rural areas such as the Lancashire and Cumbria region. However, over a 5-10 year timescale with uptake of the screening interventions the incidence of ruptured AAA should reduce to very low levels, with improved patient outcomes, cost savings and length of stay reductions.

A completely new vascular surgery training curriculum has been developed to train forthcoming modern vascular surgical interventionalists in imaging techniques, endovascular imageguided procedures and open vascular surgical procedures, but not general surgical procedures. This training structure, however, will not be implemented until formal ratification of vascular specialty status is confirmed, which is currently likely to commence in summer 2013 at the earliest, with a six-year training structure, thus providing consultants from 2019, who will then be able to perform high volumes of these procedures in suitably equipped centralised units. This training structure has thus contributed to the desire to reduce the number of centres actually performing inpatient arterial interventions, thus consolidating training experience and opportunities.

\section{VASCULAR INTERVENTION CENTRES}

The Vascular Society recommendations envisage a reduction in vascular intervention centres to a maximum of 50 centres serving the whole UK population. These centres should provide world-class modern services to reduce death, stroke and limb loss from vascular conditions. They will provide daily elective inpatient vascular surgical and interventional procedures, daily dedicated emergency vascular theatre access with appropriately trained specialist consultant surgical, anaesthetic and radiological staff, adequate intensive care and high-dependency facilities and staffing, training of new vascular surgical interventionalists, improved opportunities for research, training of specialist ancillary staff, and rehabilitation of amputees. Some vascular surgical conditions mandate emergency intervention, but many require urgent scheduled intervention which can thus be scheduled and allow optimisation of patient co-morbid medical conditions prior to undergoing the actual vascular intervention, and provision of specialist ancillary services after the procedure. The staff from the intervention centres, however, will also be responsible for providing the vascular service across the region of the population, with local delivery of as much care as possible, and specifically outpatient and day case services. This may involve a hub-and-spoke service delivery model with staff travel to local sites, and also responsibility for ancillary staff based within local provider institutions.

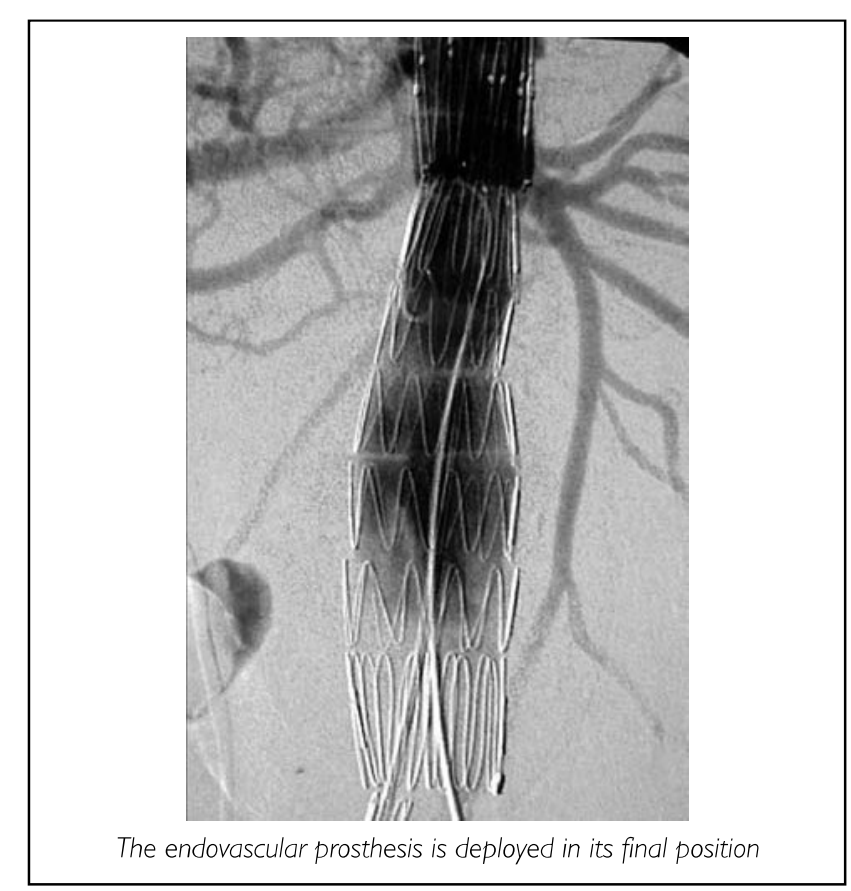

\section{IMPLICATIONS FOR PATIENTS, PUBLIC, LOCAL GPs AND EMERGENCY DEPARTMENTS}

The biggest potential risks of centralisation are a remote service with difficult access for patients, relatives and local clinicians alike - both geographically and professionally - as well as the risks of delays in emergency treatment due to increased emergency patient travel times. For the majority, centralisation of vascular services will mean that patients and visitors will be travelling longer distances. Travel times and distances to each site within Lancashire and Cumbria have been fully documented in the document entitled 'A Case for Centralisation of Vascular Services in Lancashire and Cumbria', which was submitted to the PCT Clinical Transformation Group and Collaborative Working Board. The vascular service should have mechanisms and links in place to provide transport services to the intervention centres from outlying hospitals for patient relatives (eg free shuttle bus services) and 
need to have strong public transport links and access to provision for overnight stay for patients' relatives.

There will also be concern in hospitals that are unsuccessful in retaining inpatient vascular services that other co-dependent services may be affected. The vascular network of clinicians and the intervention centre service providers have a responsibility to ensure that in addition to providing a highquality modern inpatient elective and emergency service they also provide excellent outreach services locally, both for inpatient and outpatient assessments, day case interventions and early appropriate repatriation with specialist support. Communication links should be prompt and reliable. Consideration of new technologies to aid assessments and reduce clinician (and patient) travel times should be considered, eg telemedicine in the clinical environment, radiological image links, etc. Clinical pathways should be designed which do consider the requirement for and effects on elderly patients (and relatives) with physical co-morbidities and limitations travelling long distances to centralised units, keeping these visits to a minimum.

Education and clinician engagement at local levels should also be provided, eg meetings with emergency medical staff, local GPs, other linked sub-specialties such as diabetology, stroke clinicians, rehabilitation staff, radiographers, outpatient and community nursing staff, etc.

The proposed Lancashire and Cumbria clinical model has suggested a maximum travel time of 90 minutes between selected intervention centres and acute emergency departments which no longer have on-site emergency vascular services. A consensus decision on where the actual vascular intervention centres should be located may be difficult to achieve and contentious given the nature of the geography and current secondary care services in Cumbria in particular, and the population distributions across the region. Local commissioners will need to consider these factors in addition to the acute trust submissions to provide the centralised inpatient services, and ensure that the selected centres offer good geographical coverage, equality of access for patients, yet appropriate modern facilities and staff to deliver the required improved patient outcomes which underlie the service reconfiguration.

GPs currently have an opportunity to influence this through the review process by completion of the online survey, and discussing what local provision will be required in their areas. The public may wish to be involved in any public engagement sessions held by the review management team. UHMB vascular unit clinicians continue to provide emergency and elective vascular services to South Cumbria and North Lancashire, and to that of Blackpool, Fylde and Wyre. Should local GPs or commissioners wish to discuss the review process or potential implications further with any members of our unit we will of course endeavour to make ourselves available.

Conflicts of Interest: the above article is intended to be an unbiased personal summary view of current and future proposals for vascular surgical and interventional services on a national and regional level. All details expressed within the article are within the public domain, and the article is informed by my personal involvement and participation in the following roles:

Clinical Lead for Vascular Surgical Services, University Hospitals of Morecambe Bay NHS Foundation Trust

Member of Lancashire and Cumbria Clinical Advisory Groups: Vascular Network; Stroke Network

Member of Lancashire and Cumbria Abdominal Aortic Aneurysm Screening Implementation Group

Member of North West Vascular Governance Committee

Member of The Vascular Society of Great Britain \& Ireland

Fellow of the Royal College of Surgeons of England

Contact details: mark.tomlinson@mbht.nhs.uk

Secretary: Ria Roberts: ria.roberts@mbht.nhs.uk 01524 583378 Department of Vascular and General Surgery, Ist Floor Centenary Building, Royal Lancaster Infirmary, Ashton Road, Lancaster LAI 4RP Please copy any email correspondence to my secretary for prompt attention.

\section{The Morecambe Bay Medical Journal Prize for the best article by a junior doctor/student}

A prize of $£ 200$ is awarded each year to the author of what is judged to be the best article published in the Journal.

The prize is open to all junior doctors and students. The winner of the $201 \mathrm{I}$ prize will be announced in the Summer 2012 issue of the Journal. 